\title{
PRODUCTIVITY INCREASE OF HIGH PRECISION MICRO-MILLING BY TRAJECTORY OPTIMIZATION
}

\author{
A. Schorderet ${ }^{1 *}$, R. Herzog ${ }^{1}$, N. Jacquod ${ }^{1}$, Y. Marchand ${ }^{1}$, Ch. Prongué ${ }^{2}$ \\ ${ }^{1}$ University of Applied Sciences and Arts Western Switzerland (HES-SO), HEIG-VD, Yverdon-les-Bains, Switzerland \\ ${ }^{2}$ Willemin-Macodel SA, Route de la Communance 59, 2900 Delémont, Switzerland \\ ${ }^{*}$ Corresponding author; e-mail: alain.schorderet@heig-vd.ch
}

\begin{abstract}
Milling parts for watch, medical, aircraft or molds industries is a compromise between time and precision or surface quality. The latter is very often related to machine vibrations during the process. This paper summarizes results obtained with two approaches aiming at reducing machine vibrations caused by axes accelerations. Both are control model based, taking into account the machine's modal behavior. The first algorithm optimizes the acceleration profile, while the second manages the axes cross-talk vibrations. A high-end 5-axis machine tool was used to mill $8 \mathrm{~mm}$ square pockets in brass with a $1 \mathrm{~mm}$ diameter tool and using a $0.05 \mathrm{~mm}$ depth of cut. Standard CNC parameters as well as vibration reduction options were evaluated. Then the two proposed algorithms were implemented on the same machine-tool using a laboratory Matlab based CNC. This paper describes the significant improvements provided by the algorithms when compared to a high end CNC. In the case of milling reference square pockets, up to $90 \%$ vibration amplitude reduction were achieved for a given feed rate, and a 5 -fold decrease in the pockets machining time was obtained for the same surface quality.
\end{abstract}

\section{Keywords:}

Milling; Vibrations; Trajectory optimization; Surface quality; CNC

\section{INTRODUCTION}

Production of mechanical parts by milling process for watch, aviation or aerospace industries or for the medical sector is a very demanding operation. On one side, requested dimensional and geometric tolerances are tight, and surface quality must be high. On the other hand, even if there are no large series, the manufacturing cost, related to the milling process time, is an important factor. Therefore, the machine-tools performance is defined by the precision and the machining speed capabilities. The surface quality criterion is mainly related to the machinetool vibrations during the process. This behavior being dependent on the machining speed, the accelerations and jerks required to reach this speed, there is a trade-off between productivity and surface quality.

Practically, finding the "optimal" machining parameters is usually based on a "trial-and-error" approach, from a starting point $A$ (Fig. 1) defined by experience, and from which the operator tries to reach the shortest machining time for the requested part quality criteria. If quality provided by parameters of point $A$ is not good, the satisfactory point $B$ is often found by reducing the feed speed and, by the way, the productivity. Obviously, point B should be only an intermediate point and the optimal point $\mathrm{C}$ may be reached at the limit of the capabilities (white "feasible" zone) of the hardware+software of the machine-tool. However, the requested means to reach this optimal point are not straightforward and research projects have been undertaken in this field since many years, in particular to reduce the chattering of machine-tools [Altintas 1995]. The work presented in this paper addresses the micromachining case, for which the main vibration energy is linked to the axes accelerations that therefore play a major role in the workpiece surface quality. The goal is to reduce the surface roughness patterns (wavelength $\lambda \approx 100 \mu \mathrm{m}$, depth $0.1-2.0 \mu \mathrm{m}$ ) due to vibrations in a specific micromilling case and increase the productivity.

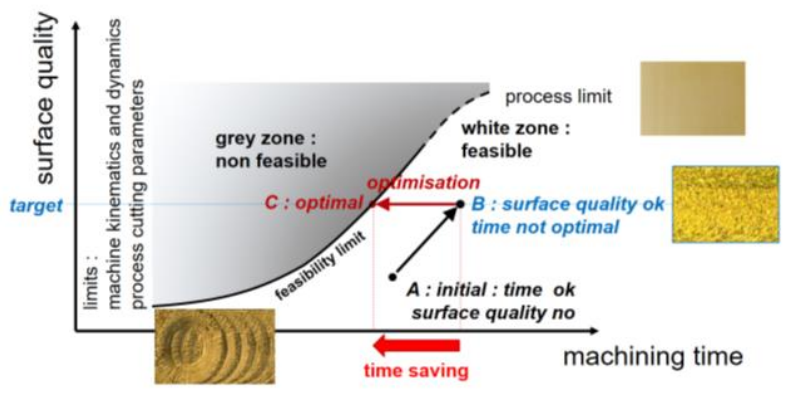

Fig. 1 Surface quality vs machining time.

The approach presented in this paper is based on a twofold improvement of the dynamic behavior, taking into account the modal behavior of the machine-tool. First, as the system energy input, the acceleration profiles are optimized. Second, a dynamic cross-talk compensation is applied to control the transient vibration amplitude. 


\section{REFERENCE MACHINING CASE}

\subsection{Micro-Milling assumptions}

Process induced machine-tool vibrations (chattering) was largely studied for more than 20 years. Several techniques and approaches aiming at chattering suppression were developed for general or specific conditions as described in [Munoa 2016]. In the present work, specific milling conditions were considered in order to address the particular industrial problem of watch main plates end milling surface quality. The process is a 2.5 axes operation (X-Y-Z) realized with a small tool ( $D \leq 1 \mathrm{~mm}), 1$ tooth, at high speed 30 '000 rpm, with depth of cut of $0.05 \mathrm{~mm}$. The workpiece material is watch-making brass EN12167. In the practice, the reference machining feedrate corresponding to this milling configuration is $\mathrm{F} 129$, providing the required surface quality. The feedrates mentioned in this paper are therefore compared to the F129 reference using a "speed factor" defined by the ratio between tested and reference feedrate. Typical watch plate is shown in Fig. 2. This kind of micro-milling process induces very low cutting forces ( $<1 \mathrm{~N}$ measured) and the tool-workpiece interface stiffness is supposed to be very low with respect to the machinetool's one [Uriarte 2007]. Therefore, the following assumption $(\mathrm{H} 1)$ can be made: the harmful vibrations occurring during considered micro-milling process are mainly caused by the machine's axes accelerations. This assumption is verified in §Chyba! Nenalezen zdro] odkazů.

\subsection{Reference pocket milling}

In order to evaluate the proposed optimization and compensation algorithms, a simple reference milling case was defined. The milling path includes combination of $X / Y$ acceleration/deceleration at each corner $\left(90^{\circ}\right.$ turn) of the "square growing spiral" depicted in Fig. 3 . For each square pocket, 5 identical turning conditions appear for each of the $4 \mathrm{X} / \mathrm{Y}$ acceleration/deceleration straight lines. Since acceleration phase is very short, only the constant speed length is varying. These kinematic conditions allow to observe surface roughness patterns depending on the acceleration (positive or negative) required by the trajectory change of direction in the corners. Fig. 4 shows two zones corresponding to accepted and unaccepted milling patterns on the reference pocket surface.

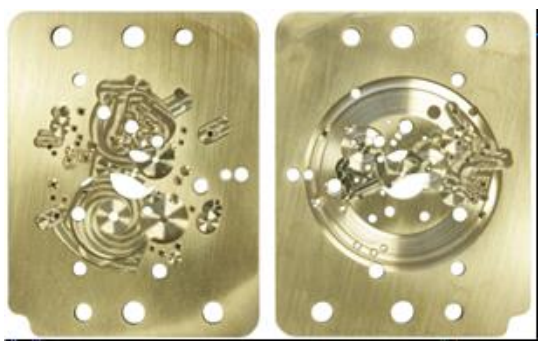

Fig. 2 Typical watch plate pocket milling.

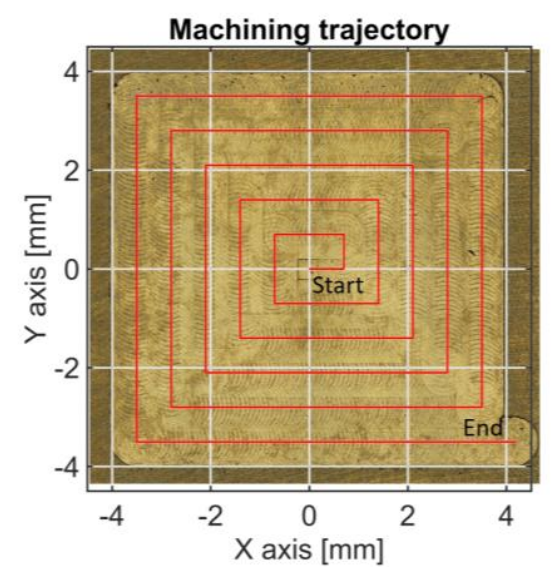

Fig. 3 Reference square pocket.

This reference pocket was repeated with different feed speeds and CNC configurations in order to compare the obtained surface quality results. A single brass bar sample (Fig 15) typically includes up to 4 different machine settings and 8 feed speeds (F127 to $F 1000 \mathrm{~mm} / \mathrm{min}$ ). Since the initial surface roughness influences the pockets final surface quality, the brass bars are prepared with the same surface finishing conditions.

\section{SURFACE ROUGHNESS AND VIBRATIONS}

\subsection{Milling patterns}

The workpiece surface quality may be evaluated using standard roughness definitions. Depending on the industry sector, qualified quality technicians are also grading the surface quality using a visual subjective evaluation. In the present work, the surface quality assessment was based on a 1 to 10 (perfect mirror) grading scheme. However, in a root-cause analysis approach aiming at linking the surface quality and the eventual source vibrations, it is necessary to define a quantitative criterion.

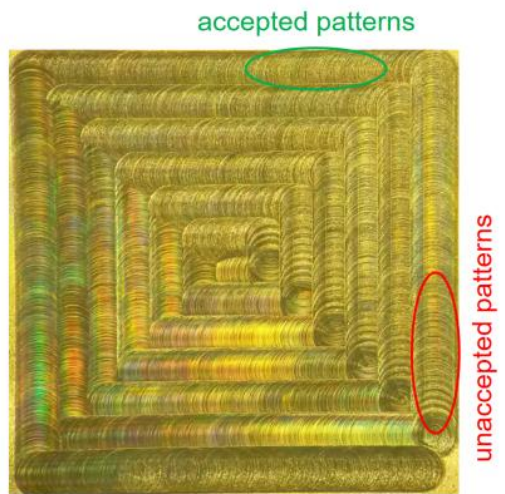

Fig. 4 Accepted/Unaccepted milling patterns: two typical regions of the pocket are shown.

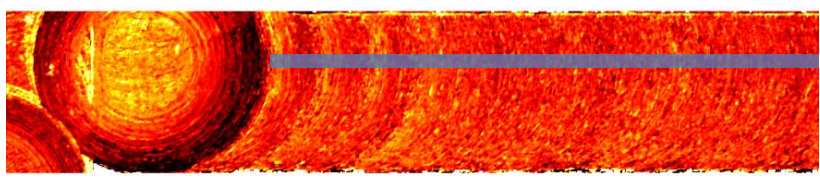

Fig. 5 Surface profile extraction line.

The surface profile is obtained using the Alicona IFM G4 microscope. For each segment of the square spiral, the surface profile extraction line is started where the back and front tooth path are superposing (Fig. 5). Then ffts of the profile are applied on $750 \mu \mathrm{m}$ portions of the segment to 
produce the corresponding spatial spectra and spectrogram (Chyba! Nenalezen zdroj odkazů.). Mean spatial frequency, corresponding amplitudes and standard deviation are then computed.

Better results were obtained in the presented test case with the spatial frequency criterion ( $4 \%$ to $6 \%$ frequency standard deviation depending on the pocket), than with the pattern method (55\% to 134\%) described in [Raphet 2008]. The criterion is defined for each pattern $i$ :

$q_{i}=\left\{\varphi_{i}, z_{i}\right\}$

where $q_{i}$ is the surface quality pattern criterion for the spatial frequency $\varphi_{i}$ of the spectrogram, and $z_{i}$ the corresponding peak-to-peak amplitude. The corresponding time frequency $f_{i}$ is obtained by multiplying $\varphi_{i}$ by the milling speed feed :

$f_{i}=\varphi_{i} v$

The spectrogram shown in Fig. 6 provides two immediate quantitative complementary elements to the visual inspection: first, the wavelength/frequency (horizontal axis) of the patterns and, second, the location where the machined "waves" appear (vertical axis). There is only one frequency that is present from the $X-Y$ corner. It starts in the short acceleration phase and its amplitude diminishes like a damped vibration in the constant speed phase.

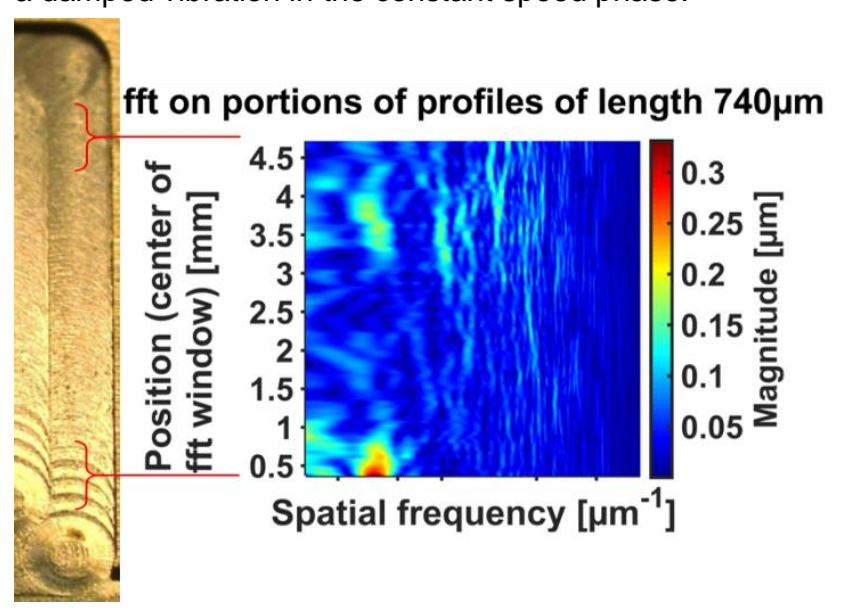

Fig. 6 Surface milling patterns and criterion Left : picture of analyzed surface Center : spectrogram with vertical axis corresponding to milled surface picture (left).

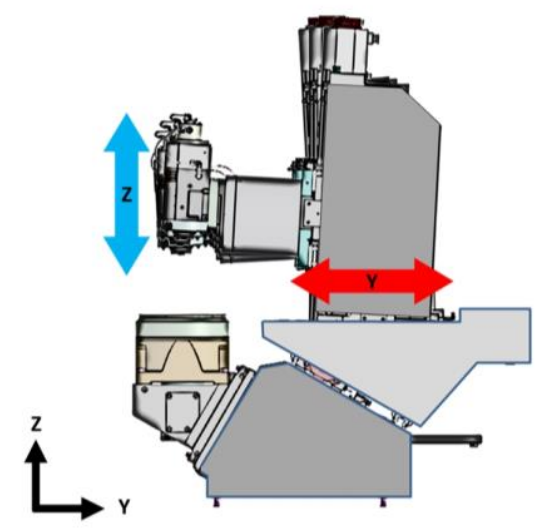

Fig. 7 Identified critical mode.

\subsection{Machine vibration modes}

The modal vibrations of the machine-tool during milling process is depending on the machine-tool's modal properties and the excitation forces. The latter being defined by the acceleration profiles and the motion control chain. Regarding the modal properties, each mode's criticality with respect to the process quality is different. Modal compliances provide an efficient mean to evaluate this criticality. Equation (3) gives the expression of modal compliance of mode number $r$ at degree of freedom (dof) number $a$ for an excitation force applied at dof number $k$.

$C_{r a k}(\omega)=\mu_{r}(\omega) \frac{p_{r a} p_{r k}}{\omega_{r}^{2}}=\frac{x_{a}(j \omega)}{F_{k}(j \omega)}$

Where $\mu_{r}(\omega)$ is the $r$-th mode dynamic amplification factor, $\omega_{r}$ is the eigenfrequency, $\boldsymbol{p}_{r}$ the mode shape, $x$ the displacement and $F$ the force.

The tool-workpiece displacement $\delta$ being directly linked to the surface quality, the corresponding modal compliance is:

$$
C_{r \delta k}(\omega)=C_{r t k}(\omega)-C_{r w k}(\omega)
$$

Modal compliance analysis allows quick identification of the most critical mode for axis accelerations forces occurring in the pocket milling process. Typical critical mode is shown in Fig. 7. For this mode, the $Y$ axis acceleration produces a large tool-workpiece relative displacement in the $Z$ direction, which is critical for surface quality. Moreover, due to the mode shape, the vibrations are not detectable on the ruler. The modal compliance may be computed from a numerical model (including damping) or from an experimental model. In the present work, FEA numerical model was updated with two experimental data sets.

First set is provided by an experimental modal analysis using an impact hammer excitation and triaxial accelerometers. The machine control-loops are active and the axes positions are set at the center of machining volume. Second set is added in order to take into account the control loop behavior at non zero-speed conditions. It focuses on the critical modes. A triangular acceleration profile is applied $\left(200 \mathrm{~m} / \mathrm{s}^{3}, 0.18 \mathrm{~g}, 444 \mathrm{~mm} / \mathrm{min}\right)$ and measurement of Tool-Workpiece displacement is realized using a RTest setup [Weikert 2004] (Fig 12). Modal frequency and damping are extracted from the constant speed phase displacement time signal. This information is used to update the numerical model by using a least squares approach. The $f_{\text {crit }}$ eigen-frequency can be directly compared to the surface quality pattern frequency $f_{i}$ obtained previously.

For confidentiality reasons, FEA model and exact modal frequencies are not given here. Likewise, the time data are not specified in the graphs of this paper. The obtained $f_{\text {crit }}$ and $f_{i}$ are very near (3.7\%), showing that the surface quality patterns (wavelength $1 / f_{\text {crit }}$ ) are caused by the identified machine-tool vibration mode at $f_{i}$.

\section{TRAJECTORY OPTIMIZATION}

\subsection{Optimization goals}

The machining surface patterns are caused by the accelerations in the corners and they show that the machine-tool's eigenmodes at $f_{\text {crit }}$ is involved. There are two ways to obtain a better surface quality with equal or increased productivity: either improve the machine's structure or the machine's control. Both ways were studied, the present paper focuses on the control solutions. Considering the information from the patterns 
spectrograms, optimization goal is to minimize the energy introduced in critical eigenmode during the acceleration phase, in order to sufficiently reduce the vibrations present in the constant speed phase (steady-state vibration phase). However, this is not sufficient and the second goal is to control the transient dynamic effect during the short acceleration phase.

\subsection{Choice of the acceleration profile}

During finishing, the main excitation source stems from the acceleration of machine axis. We consider the case of a cornering between two straight line machining trajectories, where the acceleration before and after the corner is supposed to be zero. For a corner, typically one axis must decelerate, creating e.g. $\Delta v_{x}<0$, while another axis must accelerate, creating $\Delta v_{y}>0$. Both phases may overlap, sometimes called "blend", in order to avoid standstill. More they overlap, more rounded the corner shape will be. The acceleration phases are typically short compared to the straight line travel time.

There is an infinite choice of acceleration profiles creating the same $\Delta v_{x}, \Delta v_{y}$, e.g. acceleration pulses or triangular acceleration shape. However, in order to avoid excitation of a vibration mode corresponding to a natural frequency $f_{0}$, we require the Fourier transform of the acceleration profile having a zero at frequency $f_{0}$. Other solutions involving filtering are proposed in [Altintas 2011] [Murphy 1992] [Singer 1992]. For a triangular acceleration shape, the zero in the spectrum happens if the duration is $T_{t r}=\frac{2}{f_{0}}$. Furthermore, we require the amplitude spectrum to stay low beyond $f_{0}$ in order not to excite higher modes. This frequency domain requirement translates into time domain in the sense that the acceleration profile should be very smooth, which is not the case with the triangular acceleration profile.

For this reason, we propose a "bellshaped" acceleration profile as shown in Fig. 8 on the left side. Such a signal is also known as "flat top window". It can easily be described as a sum of a constant term and 3 cosine terms, e.g. a finite Fourier series over the duration $\left[0, T_{b l}\right]$. For the determination of the four coefficients, we impose $\Delta v$, corresponding to the integral of acceleration shape, zero acceleration at the beginning and the end, the peak acceleration in the middle, and the second derivative of the acceleration (jounce) at the beginning. This linear system of four equations permits the determination of the coefficients of the Fourier series.

In order to impose a zero in the spectrum of the bellshaped acceleration profile, the duration must be $T_{b l}=\frac{4}{f_{0}}$. Fig. 8 below shows the comparison between a normalized triangular spectrum and a normalized bellshaped spectrum. Clearly, the bellshaped spectrum has a lower ability to excite higher order modes.

\subsection{Dynamic cross-talk compensation}

The choice of acceleration profile described above allows a transition from a non-excited mode @ $f_{0}$ before the corner to a non-excited mode after the corner. However, during the corner, cross talk is taking place and couples e.g. acceleration of $y$ axis to a relative displacement between tool and tool piece in $Z$ axis. Therefore, the second approach corresponds to undo this coupling by using a model-based feedforward approach. This approach is used with the Heidenhain CTC option, that only considers quasistatic coupling, as in [Thoma 2012]

To this end, we integrate the mechanical model with the servo control and feedforward loops, see Fig. 9 below.
Note that each arrow corresponds to a vector signal, and the mechanical model block incorporates dynamic coupling between the axes (cross talk). A (stable) plant inversion approach enables calculating the $z$ reference signal $w_{q z}(t)$, allowing to vanish the relative workpiece-tool position, see Fig. 10 below.

\section{EXPERIMENTAL DYNAMIC VALIDATION}

The machine-tool is a Willemin-Macodel 508S2 with a Heidenhain TNC640 CNC in the standard configuration (reference baseline case). Heidenhain options AVD and CTC are available. In the alternative configuration (called HEIG-VD) used to implement and assess the optimization algorithms, high end drives from company Triamec are utilized for axes $X, Y$ and $Z$.
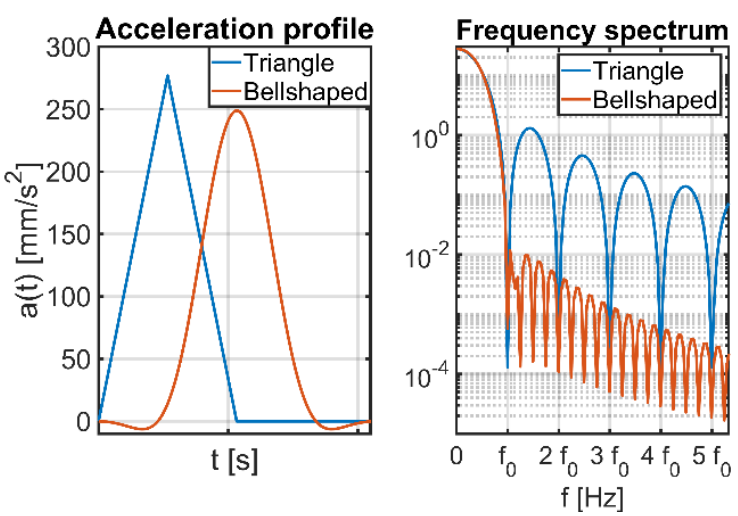

Fig. 8 Acceleration profiles and corresponding spectra for triangular and bellshaped profile.

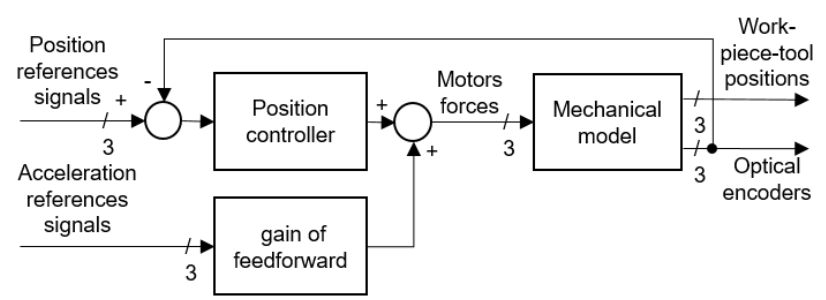

Fig. 9 Integrated Closed-loop Model

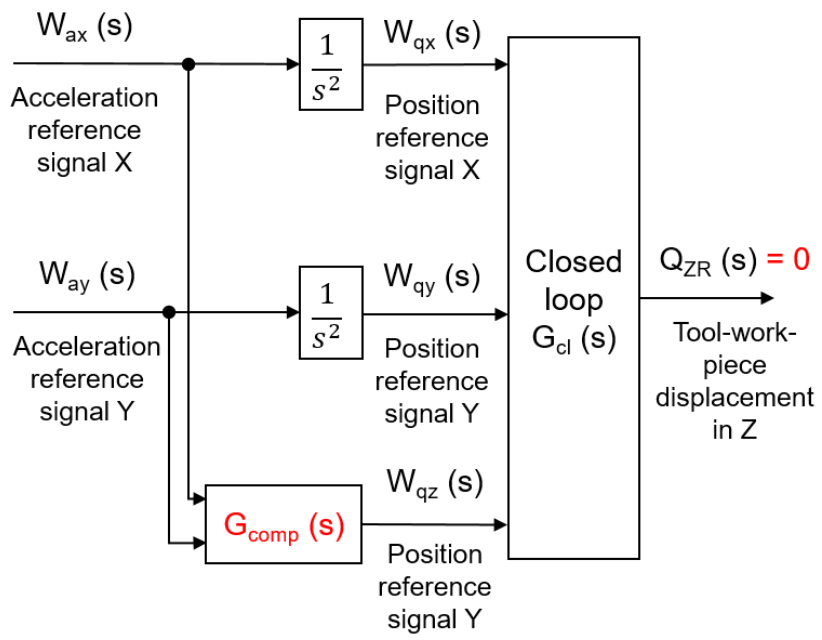

Fig. 10 Dynamic Crosstalk Compensation.

In order to compare the predicted trajectories (simulations) with effective real ones, the reference pocket machining described in Fig. 3 is first evaluated from a tool-workpiece displacement viewpoint, using the RTest setup shown in 
Fig. 11. Then, the workpieces are machined and the assessment of the surface quality is done using the $q$ criterion, and the visual inspection grading.

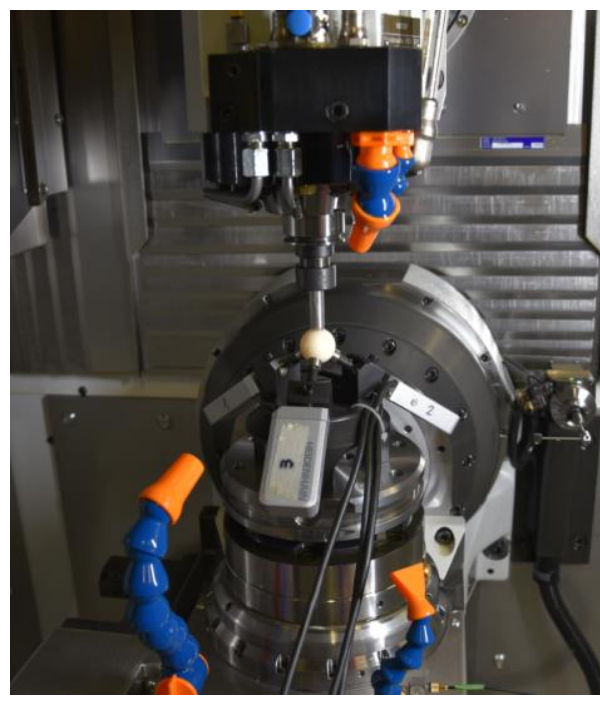

Fig. 11 RTest setup.

\subsection{RTest standard result}

The trajectories in the $X Y$ plane are defined with respect to the measured Heidenhain reference case. The exact corner trajectory is not known from this reference CNC. Therefore, $\mathrm{RTest}$ was used in a reverse engineering approach to match the reference trajectory. The obtained trajectory for the HEIG-VD CNC shows that the maximum difference in $X Y$ plane is below $3.2 \mu \mathrm{m}$ for the Triangular acceleration profile, which is the most critical The maximum contour error over the $\mathrm{F} 129$ to $\mathrm{F} 645 \mathrm{~mm} / \mathrm{min}$ speed range is 0.35 um at F444. Standard CNC is above these values.

The perturbed trajectory in the $Z$ direction are measured with RTest system for both reference HEID and HEIG-VD CNC. Results are shown in Fig 12, for the first part of a straight line. The acceleration phase is indicated by the yellow rectangle. Two values are extracted for each trajectory : $q_{z \max }$ is the maximum peak oscillation amplitude, occurring in the acceleration zone; $q_{z}$ res is the residual vibration amplitude in the constant speed zone. Tab. 1 summarizes the values obtained for feed speeds F129 to F444. One observes that the values are similar, except for F444. being $10 \%$ higher for the HEIG-VD CNC than the reference one, but the difference is only $0.2 \mu \mathrm{m}$.

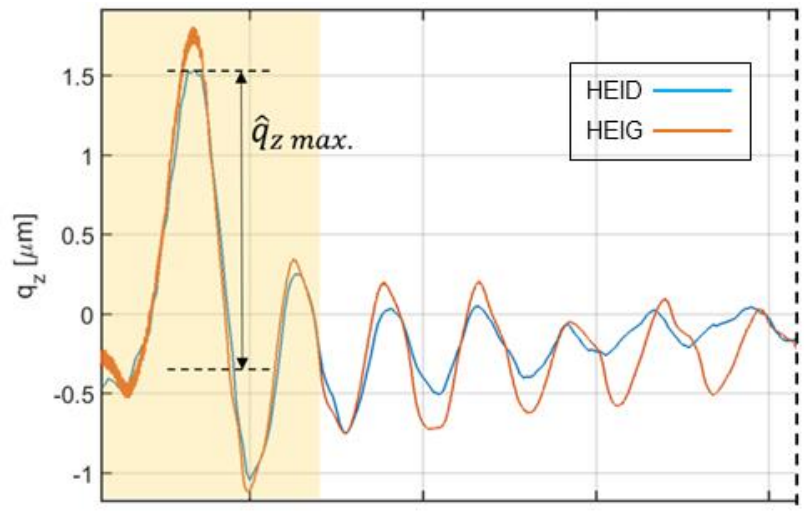

Fig. 12 R-Test comparison for standard Bellshaped at feed speed F444 - yellow part defines the acceleration phase.
Tab. 1: Comparison of Heidenhain (HEID) and HEIG-VD CNC with RTest measurements.

\begin{tabular}{|c|c|c|c|c|c|}
\hline & \multicolumn{5}{|c|}{ RTest : vibrations in Z-direction } \\
\hline & feed speed & F129 & F272 & F444 & F645 \\
\hline & speed factor & 1.0 & 2.1 & 3.4 & 5.0 \\
\hline \multirow{3}{*}{$\begin{array}{c}\hat{q}_{z \max } \\
\text { [um] }\end{array}$} & HEID & 0.61 & 1.23 & 1.81 & \\
\hline & HEIG-VD & 0.63 & 1.21 & 2.00 & 2.06 \\
\hline & rel. diff & $3 \%$ & $-2 \%$ & $10 \%$ & - \\
\hline \multirow{3}{*}{$\begin{array}{c}\hat{q}_{\text {zres }} \\
{[\mathrm{um}]}\end{array}$} & HEID & 0.11 & 0.12 & 0.11 & \\
\hline & HEIG-VD & 0.12 & 0.11 & 0.12 & 0.10 \\
\hline & rel. diff & $1 \%$ & $-1 \%$ & $1 \%$ & - \\
\hline
\end{tabular}
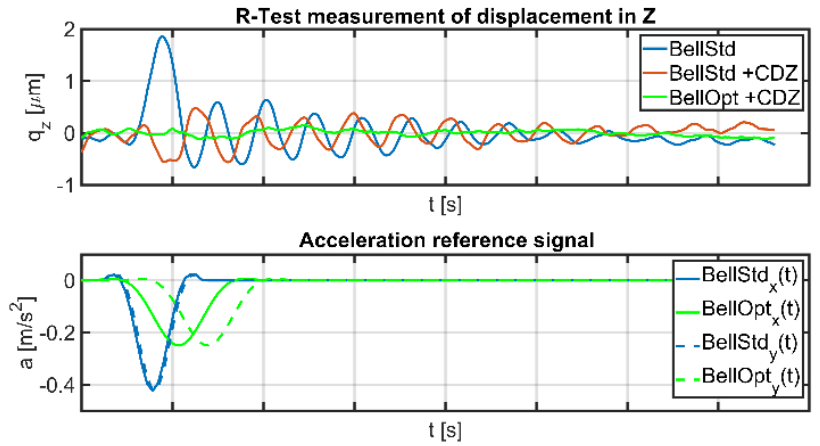

Fig. 13 Optimization type effect on Z-displacement.

\subsection{Optimization option effect}

The first comparison aims at evaluating the separate effect of each optimization type using the alternative CNC at a F444 speed. At this speed, the reference already suffers from vibrations effects. The standard profile is the bellshaped one (BellStd). On the graphs of Fig. 13, one observes that the CDZ option (dynamic cross-talk compensation of $z$ axis) reduces the transient peak of the acceleration phase, but doesn't lead to very significant reduction of the vibrations amplitudes of the steady-state phase. These vibrations start in the transient phase. However, adding the acceleration profile optimisation (BellOpt) produces a dramatic reduction of vibrations over the complete spiral segment. The acceleration profiles are also shown in Fig. 13.

\subsection{Proposed optimization vs reference and options}

The comparison of full optimized configuration and reference case describes the global improvement provided by the proposed optimized solution. The graph of Fig. 14 represents the $3 \mathrm{D}$ trajectory of the tool tip with respect to the workpiece. The RTest system's measurement zone is smaller than the pocket dimensions, only three spiral revolutions are measured. The R-Test trajectories of Fig. 14 and the milling test surface profile of Fig. 4 are compared in Tab. 3. The graph of Fig. 14 shows the very promising reduction of the vibrations amplitude achieved with the combination of acceleration profile optimization and dynamic cross-talk compensation: from $1.8 \mu \mathrm{m}^{\wedge}$ down to $0.1 \mu \mathrm{m}^{\wedge}$. 


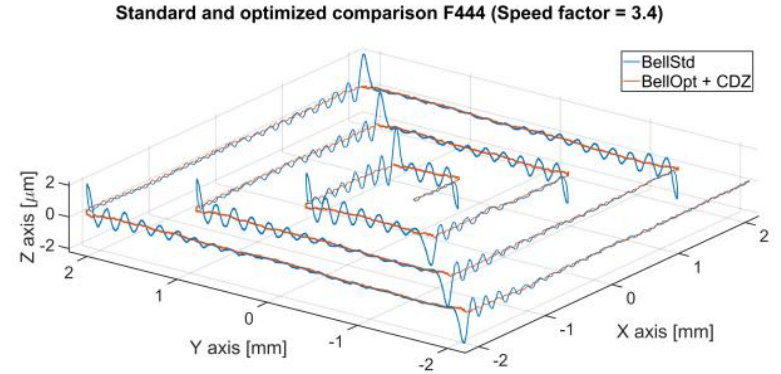

Fig. 14 R-Test comparison for standard acceleration profile (BellStd) and full optimized configuration (BellOpt+CDZ). HEIG-VD CNC.

The efficiency of the optimization algorithms over the feed speed range is shown in Fig. 15 and Fig. 16, in which the alternative HEIG-VD CNC configuration is also compared to the reference Heidenhain CNC with its CTC and AVD options. The largest z-displacements are in the acceleration phase : $\hat{q}_{z \max }$ is represented in the bar graph of Fig. 15. The corresponding relative value is computed with respect to the Bellstd case. One observes that the commercial CNC provides an improvement of $51 \%$ to $73 \%$ (F645 is unfortunately missing), with increasing speed. However, the proposed optimization provides an average $94 \%$ and still $91 \%$ at the maximum speed.

For the same cases as above, the residual vibrations in the constant speed phase are shown in Fig 16. The graph shows that the CTC + AVD options have less effect than in the transient phase: only $15 \%$ improvement are reached at F444. On the other hand, the alternative BellOpt + CDZ optimization reaches $94 \%$ at F444, average being $94 \%$. Thus, this solution provides an improvement of $85 \%$ with respect to the CTC+AVD options at F444.

The obtained results describe the tool tip-workpiece relative perturbation during the machine motion for realizing the square spiral trajectory. They show that the proposed solution is very efficient, both in the acceleration and in the constant speed phases. This alternative solution outperforms by $85 \%$ the AVD+CTC options used by the machine-tool manufacturer (average over the speed range).

The optimization solutions may introduce contour errors as described in [Altintas 2012]. Detailed analysis of contour error for all cases is not realized in this work, but will be undertaken in future. However, quick analysis of RTest results showed that the optimized trajectory deviation is below $5 \mu \mathrm{m}$.

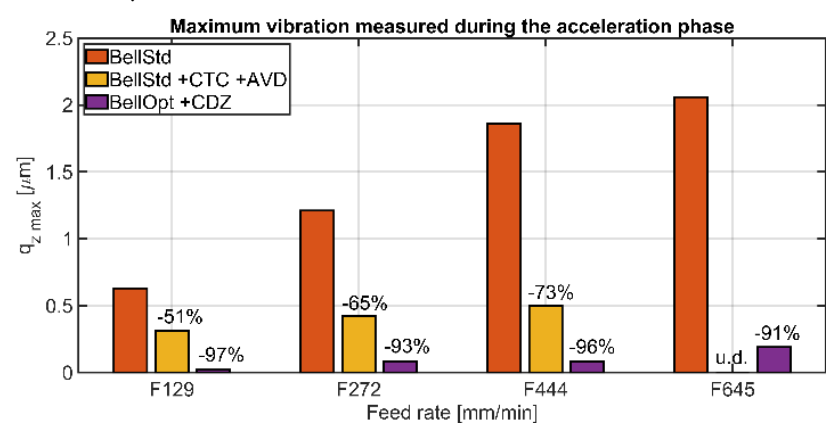

Fig. 15 R-Test results: acceleration phase.

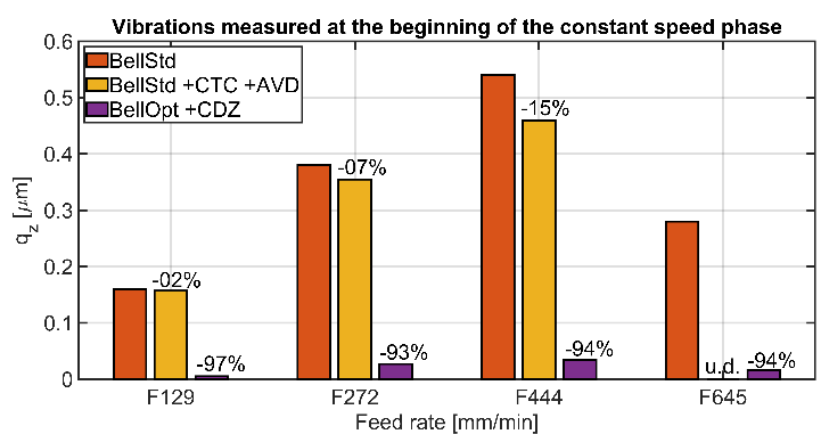

Fig. 16 R-Test results : constant speed phase.

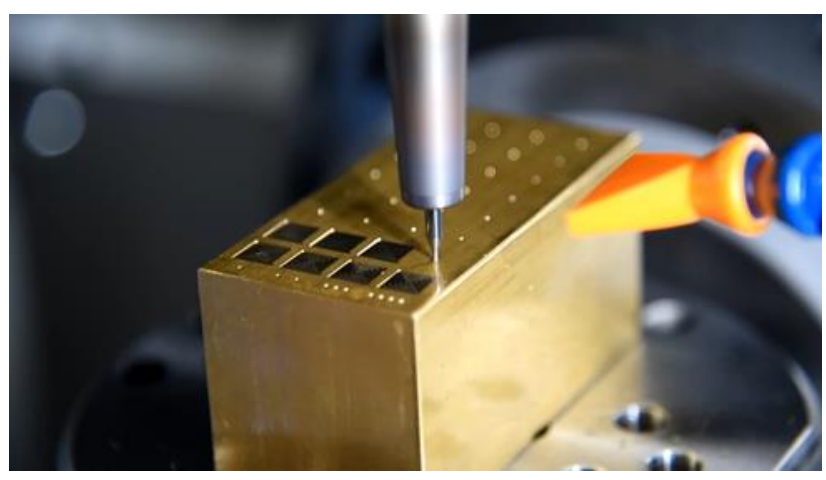

Fig. 17 Square pockets micro-milling set-up.

\section{MILLING TESTS}

\subsection{Machining conditions}

The machined workpiece is shown in Fig. 17. Tool used is a straight cut end mills, $1 \mathrm{~mm}$ diameter, 1 tooth - reinforced (Louis Bélet 111-1). Spindle speed is $30^{\prime} 000 \mathrm{rpm}$, feed speeds are from F129 to F645 mm/min. Axial depth of cut is $0.05 \mathrm{~mm}$, radial depth of cut is $0.7 \mathrm{~mm}$ ( $30 \%$ overlapping).

\subsection{Pockets microscope analysis}

The same options are tested as for the RTest dynamic trajectory measurement one. The Alicona IFM G4 microscope images of same machine paths along $Y$ axis are compiled in Fig. 18. Considering the reference case BellShaped, the BellOpt and BellOpt+CDZ pictures clearly illustrate the effect of the profile optimization on the low amplitude residual vibrations, as well as how the CDZ option almost completely erases the corner initial indentation. The BellShaped+AVD+CTC options do not reach the same visual aspect and 4 oscillations in the constant speed phase are still visible.

508S2, Heig-VD CNC, F444
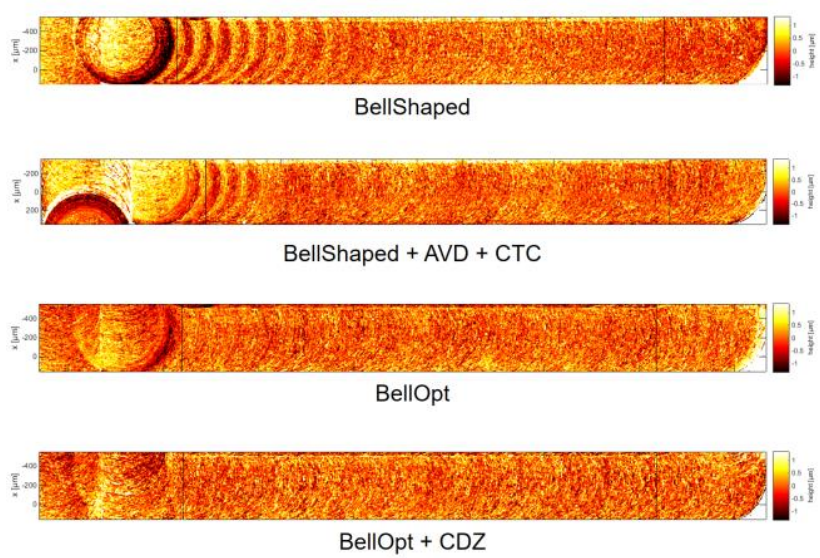

Fig. 18 Milling result for different options combinations. 
Tab. 2: Comparison of milled segments microscope analysis.

\begin{tabular}{|c|c|c|}
\hline \multicolumn{3}{|c|}{ BellOpt CDZ F444 } \\
\hline segment \# & $q_{\text {zres }}$ & $q_{\text {zmax }}$ \\
\hline 5 & 0.044 & 0.110 \\
\hline 6 & 0.031 & 0.040 \\
\hline 7 & 0.034 & 0.080 \\
\hline 8 & 0.026 & 0.130 \\
\hline 9 & 0.033 & 0.060 \\
\hline \hline average & 0.034 & 0.036 \\
\hline std dev & 0.007 & 0.036 \\
\hline
\end{tabular}

The microscope profile analysis is realized for 5 segments of the same pocket, the measurements and processing deviation is shown in Tab 2. Standard deviation values for $q_{z \text { max }}$ and $q_{z \text { res }}$ are below $0.05 \mathrm{~mm}$. Largest deviation is in the $q_{z \max }$ case, which is evaluated for a single "oscillation".

The comparison of the RTest measurement and the microscope profile analysis show a good agreement between the trajectory without milling process (RTest) and the machined profile. Tab. 3 shows that the only discrepancy appears between the $q_{z \max }$ profile value $(0.21 \mu \mathrm{m})$ and the corresponding RTest one $(0.07 \mu \mathrm{m})$. The machining process may become more important at this submicronic scale. Apart this value, the good correspondence of the machine-tool dynamic behavior with and without the process validates the assumption $\mathrm{H} 1$. The micro-milling process doesn't influence the machine's dynamics with respect to axis acceleration forces.

\subsection{Pockets visual inspection}

The pockets are inspected by Willemin-Macodel quality technician and their 1 to 10 grading scheme is applied. $\mathrm{F} 129$ is the reference speed, i.e. the surface quality target is obtained with speeds $\leq \mathrm{F} 129$. Tab. 4 shows that the alternative optimization allows to improve the surface quality at this speed with grade 8 vs 7 . The other important result is that the proposed optimization is providing equivalent surface quality up to F645 speed with grade 7 , while the reference configuration quality decreases to grade 3. This confirms the RTest measurements, and validates the proposed trajectory optimization as a significant improvement for the micro-milling.

Tab 3: Comparison of RTest and milling results.

\begin{tabular}{|l|c|c|c|c|}
\hline \multicolumn{5}{|c|}{$\begin{array}{c}\text { Oomparison RTEST - MILLING [ } \mu \mathrm{m}] \\
\text { F444 (speed factor = 3.4) }\end{array}$} \\
\hline & \multicolumn{2}{|c|}{ RTest } & \multicolumn{2}{c|}{ Milling } \\
\hline & qzres & q $_{\text {zmax }}$ & qzres & qzmax \\
\hline HEID & 0.55 & 1.86 & 0.62 & 1.54 \\
\hline HEID AVD CTC & 0.46 & 0.50 & 0.51 & 0.28 \\
\hline HEIG OPT CDZ & 0.03 & 0.07 & $<0.02$ & 0.21 \\
\hline
\end{tabular}

Tab. 4: Grading of pockets surface quality.

\begin{tabular}{|l|c|c|c|c|}
\hline \multicolumn{5}{|c|}{ Grading of pockets surface quality } \\
\hline feed speed & F129 & F272 & F444 & F645 \\
\hline speed factor & 1.0 & 2.1 & 3.4 & 5.0 \\
\hline HEID & 7 & 6 & 3 & 3 \\
\hline HEIG OPT CDZ & 8 & 7 & 7 & 7 \\
\hline
\end{tabular}

\section{SUMMARY AND CONCLUSION}

A new approach for improving micro-milling productivity and surface quality has been developed. It takes advantage of the assumption that the process doesn't influence the machine dynamics. This allows a simple and efficient approach. The relationship between the unaccepted milling patterns and the machine's modal behavior is established using profile spectral analysis and modal compliances. Dual numerical-experimental approach allows building a reliable a control model including machine-tool's dynamic mechanical model.

A two fold optimization is developed. First, optimal bellshaped based acceleration profiles avoid exciting the machine-tool's critical mode during the constant speed phase, but also during the acceleration one. The second trajectory optimization algorithm focuses on the latter phase and manages axes cross-talk vibrations with a dynamic mechanical model.

A two steps validation compared the results of a high-end CNC with the Matlab based laboratory CNC's optimized trajectories. A reference pocket milling test case is defined. First, the trajectories are measured without milling process, with an RTest system. Second, the surface quality is assessed, using an ad hoc profile analysis in conjunction with a visual inspection grading.

The results show that the new optimization algorithms provide equivalent surface quality (grade 7) up to F645 feed speed (vs F129 reference case), while the reference configuration quality decreases to grade 3 . Thus, a 5 -fold decrease in the pockets machining time is obtained with the same surface quality. Considering the vibrations amplitudes, the proposed alternative optimization also outperforms by $85 \%$ the AVD+CTC options used by the machine-tool manufacturer with the Heidenhain CNC.

These impressive results are obtained with the reference pocket and its particular growing spiral trajectory. A watch main plate milling would benefit from the developed solution. However, the proposed approach should be developed further to be applied to 3-axes and 5-axes milling of more complex industrial parts. Other materials should also be tested.

\section{ACKNOWLEDGEMENTS}

The work was supported by Innosuisse - Swiss Innovation Agency, project \# CTI 18313.1 PFIW IW

\section{REFERENCES}

[Altintas 1995] Y. Altintas, E. Budak, Analytical Prediction of Stability Lobes in Milling, CIRP Annals, Vol 44, Issue 1, 1995.

[Altintas 2011] Altintas, Y., Verl, A., Brecher, C., Uriarte, L., Pritschow, G., Machine tool feed drives, CIRP Annals Manufacturing Technology, 60, 2011

[Altintas 2012] Altintas, Y., Khoshdarregi, M.R., Contour error control of CNC machine tools with vibration avoidance, CIRP Annals, Manufacturing Technology, 61, 2012.

[Munoa 2016] Munoa, J., Beudaert, X., Dombovari, Z., Altintas, Y., Buda, E., Brecher, C., Stepan, G., Chatter suppression techniques in metal cutting, CIRP Annals Manufacturing Technology 65, 2016

[Murphy 1992], Murphy, B.R., Watanabe, I., Digital Shaping Filters for Reducing Machine Vibration, IEEE Transactions on Robotics and Automation, Vol 8, Issue 2, 1992 
[Raphet 2008], Raphet, B., Techniques de l'Ingénieur, Etats de surface-caractérisation, Réf : R1230 v2, Éditions T.I., 2008.

[Singer 1992] Singer, N.C., Seering, W.P., An extension of command shaping methods for controlling residual vibration using frequency sampling, Proceedings IEEE International Conference on Robotics and Automation, 1992.

[Thoma 2012] Thoma, S., Weikert, S., Compensation strategies for axis coupling effects. In: Elsevier, ed. Proceedings of the 5th CIRP Conference on High Performance Cutting, 2012

[Uriarte 2007] Uriarte, L., Herrero, A., Zatarain, M., Santiso, G., Lopéz de Lacalle, L.N., Lamikiz, A., Albizuri, J., Error budget and stiffness chain assessment in a micromilling machine equipped with tools less than $0.3 \mathrm{~mm}$ in diameter, Precision Engineering 31, 2007 